\title{
Dimensión ética en la organización de la atención de salud
}

\author{
GRUPO DE ESTUDIOS DE ÉTICA CLÍNICA DE LA \\ SOCIEDAD MÉDICA DE SANTIAGO*
}

Este Documento no

cuenta con Grants n

financiamiento externo. Sus autores declararon no tener conflictos de intereses.

Correspondencia a:

Dr. Carlos Echeverría B. Hospital Naval "Almirante

Nef"

Subida Alessandri s/n

Viña del Mar, Chile.

Teléfono: +56 32257

3380

E-mail: ciecheverria@ hotmail.com

\section{Organizing health care: an ethical perspective}

Health Care at population level is a complex problem. Having this in mind, the purpose of this paper is to focus on the goods that are ethically relevant in the process of caring for health at this level. We briefly analyze some of the Chilean health statistics that, although they show important improvements along the years, demonstrate that certain conditions are to be deemed as inadequate by both healthcare providers and patients. Ethics is a central component to determine how to structure and organize health care systems and how they should operate. We emphasize Human Dignity as an ethical cornerstone of the Health Care System, along with other important values such as Justice and Humanization, under the scope of the Ends of Medicine, and other components such as technical competence of providers and the financing of the whole process. We conclude that as far as a health care system is organized in a way that medical practice is well ordered, primarily and fundamentally according the Ends of Medicine and the good of persons, such a health care system is ethically adequate.

(Rev Med Chile 2013; 141: 780-786).

Key words: Ethics, Institutional; Health Planning; Health Care Quality, Access, and Evaluation.
$\mathrm{E}$ 1 cuidado y atención de salud de la población, en las sociedades contemporáneas de estructura moderna, se ha transformado en una tarea compleja y de enormes dimensiones.

Determinar los modos adecuados de organizar la atención de salud, de forma que responda a las necesidades reales de las personas, es un desafío con fuertes implicancias éticas, toda vez que se trata de acciones que afectan a las personas en sus dimensiones más significativas.

Hoy en día, en el contexto de una multiplicación y diversificación de las atenciones de salud, llevadas a cabo por personas diversas, organizadas en complejos equipos multi-profesionales, se reconoce que, más allá de la preocupación individual, resulta indispensable la participación de los ciudadanos, en orden a determinar el modo global de estructurar y ejecutar estas acciones.

Diversos estudios se han enfocado en los aspectos epidemiológicos, médicos, sociológicos, económicos y políticos que deben ser considerados a la hora de intentar proponer o ejecutar acciones de salud de alcance comunitario ${ }^{1,2}$. Sin embargo, no ha habido suficiente reflexión destinada a establecer específicamente cuáles deban ser los bienes éticos a cautelar en este orden de materias.

El presente trabajo investiga acerca de algunos bienes, valores o principios éticos que deberían ser

\footnotetext{
*Documento elaborado por el Grupo de Estudios de Ética Clínica de la Sociedad Médica de Santiago y aprobado por su Mesa Directiva (29 de abril de 2013). Integrantes del Grupo de Estudios: Dres. Jaime Burrows (Facultad de Medicina, Universidad del Desarrollo), Carlos Echeverría B. (Presidente del Grupo de Estudios; Hospital Naval "Almirante Nef", Viña del Mar), Alejandro Goic G. (Academia de Medicina del Instituto de Chile), Carolina Herrera C. (Facultad de Medicina, Universidad Andrés Bello), Carlos Quintana V. (Facultad de Medicina, Pontificia Universidad Católica de Chile), Alberto Rojas O. (Facultad de Medicina, Universidad Andrés Bello), Rodrigo Salinas R. (Facultad de Medicina, Universidad de Chile), Alejandro Serani M. (Facultad de Medicina, Universidad de Los Andes), Paulina Taboada R. (Centro de Bioética, Pontificia Universidad Católica de Chile), Ricardo Vacarezza Y. (Facultad de Medicina, Universidad de Chile).
} 
considerados a la hora de concebir e implementar soluciones de salud en el ámbito de la comunidad organizada y, en particular, en nuestro país.

\section{Consideraciones éticas y técnicas con respecto a la atención de salud en Chile}

A pesar de la complejidad que reviste el problema concreto de la atención de salud para la población y lo difícil de sus soluciones, parece necesario y pertinente desarrollar algunos criterios que permitan juzgar los componentes éticos que inciden en nuestra organización de la atención de salud.

\section{a. La situación nacional}

En Chile la preocupación de la comunidad política por estos problemas ha quedado reflejada tanto en el plano constitucional ${ }^{3,4}$ como en las instituciones públicas que han ido surgiendo. Ejemplo de esto es la fundación del Hospital San Juan de Dios, ya en 1552. Posteriormente, durante el S. XVII, se fueron desarrollando otros hospitales e instituciones de acogida tanto en la capital como en provincias. Durante el S. XIX se crearon instancias educacionales y académicas de tipo médico, surgiendo luego, en el siglo XX, numerosas instituciones de salud de alcance nacional, como el Ministerio de Salud y el Servicio Nacional de Salud, antecesor de los actuales Sistemas de Salud ${ }^{5,6}$.

Los indicadores de salud muestran, por su parte, variados logros en morbi-mortalidad tanto que el perfil demográfico de la población ha cambiado y vivimos actualmente una transición epidemiológica $^{7,8}$. En cuanto a los recursos asignados a salud, éstos son cada vez más cuantiosos y significativos 9 .

Subsisten, sin embargo, problemas en relación con la carga de enfermedades no transmisibles ${ }^{10}$, algunas inequidades en la mortalidad y acceso a las acciones de salud ${ }^{11}$, cambios en las interacciones entre médicos y pacientes ${ }^{12}$, a la vez que se han estudiado las percepciones de calidad de la atención $^{13,14}$. Adicionalmente, es posible constatar un malestar creciente en la opinión pública en relación a la organización sanitaria nacional ${ }^{15}$. Este malestar se concreta en la insatisfacción con respecto a los costos y esperas que parte de la población experimenta para acceder a una atención de salud cada vez más burocratizada, y que además manifiesta deficiencias en la calidad humana de su trato y acogida. Se hace también mención a la cuantía de las ganancias económicas de los entes financiadores de la salud privada ${ }^{16}$, las que son percibidas, en algunos casos, por parte de la población ${ }^{17}$ y por agentes de la salud como desmesuradas ${ }^{18,19}$. Además, la creciente preocupación, tanto en el ámbito público como privado, por desarrollar sistemas de atención eficientes en cuanto a la utilización de recursos materiales, ha focalizado la atención en la optimización de tipo económico, más que en la preocupación por la calidad científico-técnica de la atención, por el trato humano de los pacientes y por las adecuadas condiciones de ambiente laboral de los profesionales y funcionarios de la salud. Se da, en consecuencia, la paradoja de un sistema que ajustándose a ciertos estándares públicos y privados, parece satisfacer cada vez menos las expectativas técnicas, humanas y éticas de usuarios, funcionarios y profesionales de la salud.

Por su parte, el Ministerio de Salud ha diseñado una estrategia nacional para enfrentar éstos y otros problemas de salud del país, en las "Metas 2011-2020"20.

\section{b. Criterios éticos}

Analizamos a continuación criterios que estimamos indispensables, como marco ético, de una organización para la atención de salud.

\section{La dignidad personal}

El reconocimiento de la igual e inalienable dignidad de todos los seres humanos, sin distinción de edad, sexo, raza, estado de salud o condición social, constituye el pilar fundamental sobre el que se asienta la convivencia en la mayoría de las sociedades contemporáneas. El carácter de sujeto personal, inteligente y libre, del ser humano, ha permitido desarrollar además una compleja y articulada doctrina acerca de los derechos universales de la persona humana. En consecuencia, todo sistema sanitario deberá constituirse sobre las bases de estas conquistas éticas de la humanidad.

\section{Justicia e igualdad}

Si es claro que en términos físicos, psicológicos y sociológicos los seres humanos nos diferenciamos unos de otros, también lo es, como hemos afirmado previamente, que todos los seres humanos compartimos una igual dignidad fundamental. Lo que se ajusta a esa igualdad es 'lo justo' ético, que no es incompatible con una diversidad de condicionamientos individuales. En efecto, las des- 
igualdades naturales o legítimamente adquiridas no atentan, en principio, contra la igual dignidad fundamental de las personas. Las desigualdades que sí determinan reivindicación ética, son aquellas que comportan injusticia, es decir, tendientes a impedir que cada cual tenga lo que le corresponda, especialmente si provocadas por la acción libre de un ser humano -que podía y debía evitarlas- sobre otro.

En nuestro país, por ejemplo, se constatan desigualdades en la libertad de opción en materia de salud, no solamente desde el punto de vista de los recursos que una persona pueda comprometer en ello, sino también aquellas derivadas de factores estructurales, avalados o tolerados por la legalidad. Una permanente y mal resuelta pugna entre lo público y lo privado, hace difícil una discusión desapasionada y objetiva sobre los aportes reales que uno u otro componente del sistema aportan en grados de libertad.

Se aprecian además grandes diferencias en la infraestructura dedicada a la salud y su equipamiento a lo largo de Chile. Ello determina que, según el lugar donde vivan, personas con patologías similares no tengan acceso a recursos técnicos semejantes.

Ciertos programas de salud orientados a beneficiar a la mayoría de la población, al establecer obligaciones de oportunidad/ejecución, han determinado que otras enfermedades, no incluidas en el programa, sean postergadas en su calidad $u$ oportunidad de atención, al no haber suficientes recursos para ello.

Además, el acceso regulado a prestaciones de salud ha hecho surgir nuevas normativas, con complejos protocolos técnicos y administrativos que en ocasiones distraen la atención de los profesionales hacia la perfecta realización del trámite más que a la adecuada atención del paciente. La multiplicación de instancias de registro, verificación y control, termina erigiéndose en un obstáculo al acceso, impedimento que puede afectar más a aquellas personas con menos recursos psicológicos, culturales y económicos. Estas personas, además, suelen ser las que tienen mayor necesidad de atención.

El desigual acceso a medicamentos e insumos médicos obliga a tomar decisiones que muchas veces terminan resolviéndose, tanto en el ámbito público como privado, por la vía del menor precio, sin prestar suficiente atención a la calidad. Un intento de mejorar esta situación corresponde a la creación de una agencia nacional de medi- camentos, orientada a ocuparse de la calidad y bioequivalencia de los mismos ${ }^{21}$.

Las diferencias en disponibilidad de profesionales y especialistas calificados, junto a la implementación de algunas lógicas de corte más mercantil que médico, han generado la formación de unidades asistenciales que no siempre cuentan con personas que posean los conocimientos, habilidades y experiencia específica para resolver asuntos que su promoción comercial ofrece como presentes. A su vez, una regulación del trabajo médico, tanto de profesionales como de personal paramédico, que se focalice sólo en la eficiencia económica tiende a reducir el reposo necesario para un trabajo técnica y humanamente adecuado.

Los ejemplos señalados apuntan a que la organización de la atención de salud en nuestro país sufre de desigualdades, que se han generado y desarrollado en el seno de nuestra historia, lo que implica que, en justicia, somos solidariamente responsables de las mismas, en tanto que miembros de una comunidad organizada.

Junto con el surgir contemporáneo de una mayor conciencia colectiva acerca de la responsabilidad de hacerse cargo de una organización justa y humanitaria de la atención de salud, emerge también en las sociedades modernas una reivindicación acerca de un pretendido 'derecho a la salud'. La determinación del contenido preciso de un tal derecho tiene complejidades y se ha prestado para generar un clima de exigencias, no exento de hostilidades, entre beneficiarios y prestadores de las atenciones de salud. Una responsabilidad significativa cabe en esto a la imprecisa y excesivamente ambiciosa definición de salud, propuesta por la $\mathrm{OMS}^{22}$. En efecto, si se considera que el contenido de este derecho a la salud debe estar regulado por esa definición, se hace caer sobre los hombros de las comunidades políticas una obligación que no están en condiciones de satisfacer. No parece entonces razonable confundir una aspiración de algunos ciudadanos con un derecho político concreto, susceptible de ser exigido.

En esta misma línea, crítica a la existencia de un derecho a la salud, el bioeticista estadounidense Leon R. Kass, como lo cita Van Spijk ${ }^{23}$, ha hecho notar que: "No es más lógico reclamar un derecho a la salud que un derecho a la sabiduría o al coraje. Estas excelencias del alma y el cuerpo requieren un don natural, atención, esfuerzo y disciplina de parte de cada persona que los desea. Convertir mi salud en 
el deber de algún otro, no sólo no es justo sino que impone un deber imposible de cumplir... La teoría de un derecho a la salud se escapa del buen sentido, sirve para minar la responsabilidad personal, a la vez que coloca obligaciones donde no son útiles ni posibles de cumplir".

Parece más correcto, en consecuencia, la postulación de un derecho político al acceso a una atención de salud (como es recogida en nuestra Carta Fundamental) que sea eficiente, justa y humanitaria, relativo a las posibilidades históricas concretas de cada comunidad. Una tal obligación parece más susceptible de ser llenada de un contenido preciso y de ser evaluada en su realización.

Lo anterior debe comprenderse sin perjuicio de las responsabilidades que tiene cada persona con respecto al cuidado de su salud, adoptando las medidas preventivas propias de una vida sana, más aquellas que deriven de una correcta aplicación de los tratamientos pertinentes y oportunos cuando se presente una enfermedad.

\section{Humanización en la atención de salud}

La insatisfacción por relación a la calidad ética de nuestra organización sanitaria no parece depender solamente de la constatación de situaciones de injusticia, sino también con la pérdida de otros valores que intuitivamente se consideran como esenciales en la atención sanitaria. Se suele englobar a esta serie de bienes, principios o valores, conexos entre sí, bajo expresiones generales como 'solidaridad' o 'benevolencia'. La solidaridad tiene que ver con la posibilidad de alcanzar ciertos bienes, cuando los miembros de una comunidad se hallan unos con otros 'sólidamente' unidos, y con la percepción que frente a ciertos objetivos comunitarios es este modo solidario el que se debe privilegiar.

La benevolencia, por otra parte, alude a disposiciones afectivas adquiridas, que los miembros de una comunidad libremente se regalan entre sí, y que se manifiestan de múltiples formas como por ejemplo: la cortesía entre ciudadanos, la compasión, el servicio desinteresado, el voluntariado, la beneficencia organizada y otras.

Ligada a estas disposiciones se encuentra la denominada 'vocación de servicio' que suele ser considerada por el ciudadano común como una cualidad particularmente deseable en un profesional de la salud. Ella va más allá de la simple prestación material de un servicio, al realizar una acción técnica y humana que, además, está imbuida de una actitud de compromiso y acogida con el enfermo.

La caridad, por su parte, entendida como amor desinteresado por el semejante que sufre, ha jugado históricamente un rol fundamental en la historia sanitaria de Occidente, ya sea en su dimensión cristiana de manifestación del amor de Dios en el mundo, ya sea como simple expresión de nobleza moral y de amor entre los seres humanos. De este modo, cuando se percibe que la atención de salud en un país está deprivada de estas motivaciones se la experimenta y se la juzga certeramente como 'deshumanizada'.

Con respecto a la ausencia progresiva de valores ligados a la benevolencia que, a veces, se constata en el personal médico y administrativo y en el modo de pensar y ejecutar la atención de salud en nuestro país, se suelen indicar diversos responsables.

De entre ellos, uno de los más frecuentemente incriminados en la actualidad es la incorporación progresiva de criterios de orden económico en la toma de decisiones en la atención de salud. Para tener una apreciación ecuánime de este problema es necesario reconocer en primer lugar el carácter inevitable de esta introducción. En efecto, la llamada 'masificación' en el acceso de la población a ciertos bienes públicos o privados, anteriormente no posible para una cantidad significativa de personas, exige criterios de manejo contable y de conocimiento, de experiencia y de oficio económico que los profesionales de la salud, en tanto que tales, salvo estudios y entrenamiento específicos, no solemos poseer. Esta introducción de criterios de racionalidad económica, y de racionalidad social, llamada 'de políticas públicas', ha significado para el personal sanitario un enorme y difícil esfuerzo de cambio cultural que, sin lugar a dudas, ha tenido efectos positivos en algunas áreas de la salud. No obstante lo anterior, los problemas se han generado cuando los criterios de racionalidad social o económica se han sobreimpuesto a los criterios de racionalidad médica y ética, en lugar de subordinarse a ellos, generando tensiones, frustraciones y malestar, tanto en el personal médico y administrativo, como en los beneficiarios de la atención de salud.

\section{Atención a los fines propios de la medicina}

En lo que respecta a la organización de la atención de salud se puede afirmar que cuando la racionalidad social o económica se pone al servicio 
de la mantención y recuperación de la salud, y de los valores éticos que operan en ella, tanto en lo individual como en lo colectivo, se da un orden que potencia tanto al arte médico y sus finalidades, así como al bienestar humano, la paz social y la concordia entre ciudadanos. Cuando la racionalidad política o económica se desorienta o se autonomiza, y se dirige sea sólo al beneficio económico de unos pocos, o a la reducción sistemática e injustificada del gasto público, o al despilfarro, o meramente se pone al servicio de las ideologías políticas, se produce un desorden que genera decaimiento en la calidad científico-técnica de la medicina, descontento por parte de los beneficiarios, desaliento y frustración de parte de los profesionales, como alteración de la concordia y de la paz social.

Una organización de la atención de salud, para ser éticamente aceptable, debería ser capaz de reconocer a la medicina como un bien, que busca sanar, mejorar y/o rehabilitar a los enfermos cuando es factible, a la vez que acoger y ayudar siempre, con conciencia de las dificultades sociales y económicas que enfrentan el diseño y puesta en práctica de las complejas acciones de salud de la actualidad. Profesionales de la salud, del ámbito económico y financiero, juristas, legisladores, administradores públicos y privados, como también otros que se precisen, debieran ser capaces hoy en día de entrar en diálogo constructivo, que permita encontrar soluciones médicamente satisfactorias, respetuosas de la dignidad de las personas en toda instancia y nivel; que sean social, jurídica y económicamente factibles, a la vez que convenientes y aceptables para la población.

\section{Capacidad técnica}

Es claro que la posibilidad de acceder a una adecuada atención de salud depende significativamente de la existencia de una infraestructura apropiada y un grupo humano con condiciones técnicas, habilidades específicas, cualidades personales y prestancia moral que operen debidamente los sistemas de salud.

Considerando el valor de la vida humana, en tanto bien humano básico ${ }^{24}$ como que, acorde con Gómez-Lobo, "para la bioética los bienes humanos que juegan el papel de punto arquimédico son la vida y la salud"25, se le exige a quienes participan profesionalmente en el área de la salud condiciones de alto nivel de perfección, tanto en lo personal como profesional.

\section{El financiamiento}

El financiamiento de la atención de salud de una comunidad puede concebirse como un compromiso entre dos extremos, ya sea un financiamiento estatal total, ya sea un sistema exclusivamente financiado por privados. En realidad se trata casi siempre de sistemas mixtos que se inclinan en uno u otro sentido.

En los últimos años ha surgido una fuerte conciencia crítica en nuestro país que cuestiona el carácter 'no solidario' de la estructura del financiamiento sanitario. Se suele, además, atribuir este supuesto defecto a la coexistencia en Chile de dos grandes sistemas de financiamiento y de atención de salud: el público y el privado. Mucho se ha insistido en las diferencias entre recursos y cargas de ambos sistemas; sin embargo, las discusiones en torno al juicio ético que corresponda a esta situación adolecen de falta de rigor racional y de serenidad, debidas a la frecuente adscripción de los disputantes a esquemas ideológico-políticos que tienden a ser intolerantes con quienes piensan en contrario. Parece necesario que se generen en el país instancias de reflexión que permitan abordar este tema de manera suficientemente libre, respetuosa y con el rigor intelectual que se le debe pedir a la búsqueda de soluciones a problemas que afectan, de manera vital, a todas las personas.

Es también relevante considerar que los financiamientos que se otorguen a diversos programas de salud deben ser coherentes con la finalidad que se persigue y tener la duración y sustentabilidad temporal que aseguren su permanencia. Desgraciadamente en ocasiones se realizan asignaciones con criterios de satisfacción de clientela política, otorgando recursos parciales a muchos programas que se financian, sin embargo, de modo incompleto. Esta venalidad e ineficiencia, en el uso de recursos que son comunes, constituye una flagrante injusticia que, como siempre, afecta más a los que menos tienen.

\section{El conocimiento, la formación y entrenamiento profesional}

La búsqueda, generación, obtención y difusión del conocimiento en las ciencias relacionadas con la salud y aquellas que pueden dar sustento auxiliar a la administración de los recursos utilizados, deben constituir una preocupación significativa en los sistemas de salud. Sin embargo, las características y amplitud de esos temas exceden las 
posibilidades de su desarrollo en este trabajo.

La complejidad y extensión de los conocimientos, destrezas y habilidades hoy indispensables para una correcta atención profesional plantea exigencias éticas colectivas. No sería justa ni honesta consigo misma una comunidad que descuide la formación de sus profesionales.

\section{Conclusiones}

En menos de cien años nuestra comunidad política ha experimentado, en los más diversos campos, inmensas transformaciones. La atención de salud individual y comunitaria no ha sido ajena a estas vicisitudes. El desarrollo científico-técnico, político e institucional de nuestra nación y de nuestra organización para la atención de salud ha redundado en una mejora significativa en los indicadores sanitarios de la población. No obstante lo anterior, el modo de organizarnos para atender a las necesidades de salud de nuestros semejantes adolece de deficiencias relevantes de carácter ético, tanto de justicia como de carácter humanitario.

Es posible apreciar un claro progreso histórico en la conciencia nacional en cuanto a reconocer una responsabilidad colectiva sobre la calidad y la equidad de la atención sanitaria. Esta mayor conciencia política se ha visto reflejada tanto en los textos fundantes de nuestra vida social y en la creación de instituciones nacionales de salud, como también en la mejoría objetiva de indicadores sanitarios generales.

La relativa ausencia de un análisis crítico de carácter específicamente ético sobre los modos generales de organizar la atención de salud comunitaria ha dificultado la toma de conciencia acerca de estas carencias y ha demorado la posibilidad de subsanarlas. Nuestro esfuerzo ha estado encaminado a proveer algunas de estas herramientas conceptuales que faciliten el diagnóstico y hagan más factible la implementación de una terapéutica racional.

Pensamos que nuestro sistema de organización de la atención de salud adolece de deficiencias en ámbitos como el respeto a la dignidad del que sufre, de la justicia, de la honestidad, de la solidaridad, de la caridad, de la vocación de servicio y de otros. Estas deficiencias inciden de modo muy significativo en la insatisfacción que manifiestan los beneficiarios actuales o potenciales de la atención de salud, en la frustración que experimentan los profesionales sanitarios y administrativos, y en las reivindicaciones de orden social y político. Está en nuestro poder tener la voluntad de avanzar en estos temas. Estimamos que nuestra comunidad política tiene una oportunidad histórica de dar un salto de calidad en esta materia. El hacerlo o no depende fundamentalmente de nuestro libre querer y no de implementaciones previas de orden financiero o instrumental.

Pensamos que una manera de organizar la atención de salud que permita que el arte médico se ordene, de modo primario y fundamental, a la consecución de sus finalidades propias y al bien de la persona, es éticamente adecuada. Si la organización y/o el profesional de la salud priorizan su bienestar económico, su prestigio profesional o científico, o la satisfacción de sus propias necesidades emocionales por sobre el bien físico y moral de los pacientes que requieren de sus cuidados, el ejercicio profesional se desordena, el paciente se siente instrumentalizado y se daña el espíritu de trabajo del equipo de salud.

Para que estas organizaciones tengan una real existencia en la práctica, la medicina y profesiones relacionadas deben ser enseñadas a quienes estén intelectualmente capacitados y evidencien poseer las disposiciones humanitarias propias de la profesión o se comprometan a desarrollarlas. Los que la aprendan deben ser además confrontados con sus responsabilidades y obligaciones morales, comprometiéndose a cumplirlas de modo formal y público, tal como lo señalan los preceptos y valores del Juramento Hipocrático. De esta manera se reconoce explícitamente la inspiración beneficiente y humanitaria de las atenciones en salud, que tan profundamente deben unir a enfermos y prestadores sanitarios.

Es necesario que la firme voluntad de no dañar en sus derechos a nuestros conciudadanos se manifieste, por ejemplo, en los criterios que se usan para asignar recursos. Estos criterios deberían fundarse en un conocimiento adecuado de la diversidad de realidades geográficas, culturales y sociales; ajustarse a las necesidades reales de las personas; a la vez que deberían ser conocidos por todos y eventualmente sometidos a debate público.

Dada la inevitable desigualdad de la riqueza y la diversa afectación por la enfermedad, parece evidente que, dejados a la merced de sus propios recursos, los ciudadanos tendrían un inequitativo acceso a la salud, pudiendo algunos subvenir a sus 
necesidades y otros quedar fuera de esa posibilidad. Así, se han desarrollado diversas estrategias para que aquellos sin acceso oportuno a la atención de salud lo logren, a través de una inteligente y respetuosa aplicación de la justicia distributiva. Cuán eficientes y justas han sido estas estrategias es un tema que en nuestro país no está completamente aclarado, aunque es evidente que en términos de justicia todavía queda mucho camino por recorrer. Sería muy deseable que en Chile estas evaluaciones fuesen posibles de llevar a cabo con seriedad, ecuanimidad, libertad, ausencia de dogmatismos y prejuicios, y con voluntad benévola y solidaria.

\section{Referencias}

1. Denis JL, Lamothe L, Langley A, Guerard S. Reforma y gobernanza en salud: el atractivo del gerenciamiento en la acción pública (Governance and Health: The rise of the managerialism in public sector reform). Rev Salud Pública 2010; 12 (1): 105-22.

2. Castillo-Riquelme M, Zárate V. (eds.) Evaluaciones económicas de intervenciones en salud. Rev Med Chile 2010; 138 (Supl 2): S 65-S 108.

3. Constitución Provisoria para el Estado de Chile, 1818. Capítulo VI, Artículo 2º.

4. Constitución Política de la República de Chile. Capítulo III, Artículo 9o.

5. Molina C. Historia de la Protección Social de la Salud en Chile: 1938-1973. En "Historia de la Protección Social de la Salud en Chile". Leandro Urbina Ed. LOM Ediciones, Santiago de Chile, 2009. pp. 60-84.

6. República de Chile. Ley 10.383 , de 08 de agosto de 1952.

7. Instituto Nacional de Estadísticas. Chile. Chile: Proyecciones y Estimaciones de Población. 1990-2020. País y Regiones. Disponible en: http://palma.ine.cl/demografia/ menu/EstadisticasDemograficas/DEMOGRAFIA.pdf (consultado el 20/04/12).

8. Szot J. La transición demográfico-epidemiológica en Chile, 1960-2001. Rev Esp Salud Pública 2003; 77 (5) 605-13.

9. Illanes JP. (Coordinador Ejecutivo). Informe Comisión Presidencial de Salud. Chile, diciembre de 2010.

10. Departamento de Salud Pública, Escuela de Medicina, Pontificia Universidad Católica de Chile. Informe Final. Estudio de Carga de Enfermedad y Carga Atribuible. Julio de 2008.

11. Mardones F. Algunos antecedentes sobre la inequidad en la situación de salud del adulto mayor en Chile. Rev Med Chile 2004; 132: 865-72.
12. Horwitz N. El cambio de la práctica médica. Desafíos psicosociales para la profesión. Rev Med Chile 2004; 132: 768-72.

13. Castillo L, Dougnac A, Vicente I, Muñoz V, Rojas V. Los predictores de satisfacción de pacientes en un centro hospitalario universitario. Rev Med Chile 2007; 135: 696-701

14. Riveros J, Berne C, Múgica JM. Gestión y satisfacción en servicios de salud de Chile: Contraste entre las percepciones de los funcionarios y los usuarios. Rev Med Chile 2010; 138: 630-8.

15. Superintendencia de Salud. Chile. Estudio Opinión Usuarios del Sistema de Salud. Santiago, marzo de 2011. Disponible en: http://www.supersalud.gob.cl/documentacion/569/articles-6953_recurso_1.pdf Consultado el 05 de junio de 2012.

16. Superintendencia de Salud. Chile. Utilidades Sistema Isapre 2011. Disponible en: http://www.supersalud.gob. cl/568/w3-article-7523.html Consultado el 6 de junio de 2012 .

17. Isapres: Polémica por ganancias y solidaridad. Revista Ercilla N ${ }^{\circ} 3.287$, del 13 al 26 de febrero de 2006.

18. Paris E. Estado y Salud: El comienzo de gobierno de la Dra. Michelle Bachelet. Cuad Med Soc (Chile) 2006; 46 (1): 3-4.

19. Rosas N. Presidenta Regional de Fenpruss cuestiona ganancias millonarias de las Isapres y privatizacion del sistema de salud chileno. Disponible en: http://www. radiosantamaria.cl/rsm/noticias/4222-presidentaregional-de-fenpruss-cuestiona-ganancias-millonariasde-isapres-y-privatizacion-del-sistema-de-salud-chileno. html, consultado el 06 de junio de 2012.

20. Ministerio de Salud. Chile. Metas 2011-2020. En: http:// www.minsal.gob.cl/portal/docs/1/5648324.pdf, consultado el 11 de diciembre de 2011.

21. Instituto de Salud Pública. Chile. Resolución 334/2011. Disponible en: www.ispch.cl/sites/default/files/resolucion/2011/03/Resol\%20334\%20refundida_0.pdf, consultado el 06 de junio de 2012.

22. Organización Mundial de la Salud. En: www.who.int/ governance/eb/who_constitution_sp.pdf, consultado el 06 de noviembre de 2011.

23. Van Spijk P. Aspects of the WHO definition of health. En: Person, Society and Value. Edited by P. Taboada, K.F. Cuddeback and P. Donohue-White. Kluwer Academic Publishers, Dordrecht, The Netherlands, 2002. pp. 20927 (traducción personal).

24. Gómez-Lobo A. Los bienes humanos: Ética de la ley natural. Mediterráneo, Santiago, 2006.

25. Gómez-Lobo A. Fundamentaciones de la Bioética. Acta Bioethica 2009; 15 (1): 42-5. 